\title{
Safety of Intravenous Thrombolysis for Stroke in a Patient With Multiple Intracranial Neoplasm
}

\author{
Ashkan Mowla, ${ }^{1,}$ Navdeep Singh Lail, ${ }^{1}$ Karanbir Singh, ${ }^{1}$ Sandhya Mehla, ${ }^{1}$ and Peyman Shirani ${ }^{1}$ \\ ${ }^{1}$ Department of Neurology, State University of New York, Buffalo, New York, United States \\ "Corresponding author: Ashkan Mowla, Department of Neurology, Gates Vascular Institute, State University of New York (SUNY), 100 High Street, Buffalo, New York, United \\ States. Fax: 716-8592430, E-mail: mowla_a@yahoo.com
}

Received 2015 September 05; Accepted 2015 October 06.

Keywords: Safety, Intravenous Thrombolysis, Stroke, Intracranial Neoplasm

\section{Dear Editor,}

Intracranial neoplasm (IN) are currently considered as a contraindication for intravenous (IV) thrombolysis in acute ischemic stroke (AIS) patients due to a hypothetical potential increase in the risk of intracranial hemorrhage (ICH) and based on expert opinions (1).

An 83-year-old woman developed sudden onset rightsided hemiparesis and aphasia. She presented to another hospital in our region within the first 3 hours after symptom onset and was found to have disabling neurological deficits with a national institute of health stroke scale (NIHSS) of 19. initial non-contrast head computed tomography (NCHCT) at the other hospital was read as "no acute abnormality". We only had access to the report of the initial NCHCT and were not able to review the image. She had no obvious contraindication for IV recombinant tissue plasminogen activator (rtPA). She received IV rtPA based on the standard protocol 1 and afterwards, she was transferred to our medical center for higher level of care. She arrived in our emergency department 6 hours after the symptom onset when she was found to have improvement in her language function and her NIHSS decreased to 14. A NCHCT was done right after she got to our hospital which did not show any evidence of ICH but showed a $1.4 \mathrm{~cm}$ midline hyperdense mass suggestive of a planum sphenoidale meningioma. Brain Magnetic Resonance Imaging (MRI) with and without gadolinium was performed 6 hours later which demonstrated acute infarcts in the left frontal lobe. In addition, brain MRI revealed 2 meningiomas. There was a $2.2 \mathrm{~cm}$ homogeneous enhancing mass of the planum sphenoidale, compatible with meningioma. Additionally, there was a small $0.9 \mathrm{~cm}$ enhancing nodule involving the left frontal convexity, compatible with meningioma (Figure 1). NCHCT was repeated in 24 hours after IV rtPA administration which did not show ICH or hemorrhage within the neoplasms. Within next 48 hours, NIHSS improved to
9 and then to 4 on the day of discharge.

A computerized search in Medline, Pubmed and Google scholar till April 2014, revealed only 8 cases reported of IV thrombolysis in patients with benign Ins (2-5).

Four were for AIS, 3 for myocardial infarction and one for a suspected stroke, which turned out to be a low-grade astrocytoma. Of the 4 cases that received IV rtPA for AIS, one had an acoustic schwannoma and 3 had a meningioma with no ICH post IV rtPA(2-4). Among the 3 patients who received IV thrombolysis for myocardial infarction, one had a pituitary adenoma and 2 had one meningioma each, neither developed ICH post IV thrombolysis (5).

To the best of our knowledge, this is the first reported patient of AIS who received IV rtPA and had more than one IN, both were meningiomas. Our case confirms the previous literature that the presence of benign IN does not increase the risk of ICH in AIS patients who receive IV rtPA.

\section{References}

1. Jauch EC, Saver JL, Adams HP, Bruno A, Connors JJ, Demaerschalk BM, et al. Guidelines for the early management of patients with acute ischemic stroke: a guideline for healthcare professionals from the American Heart Association/American Stroke Association. Stroke. 2013;44(3):870-947. doi: 10.1161/STR.ob013e318284056a. [PubMed: 23370205].

2. Hsieh $\mathrm{HC}$, Chen $\mathrm{CH}$. Tissue plasminogen activator use in a patient with acute ischemic stroke coexisting with meningioma. Clin Neurol Neurosurg. 2009;111(6):562-3. doi: 10.1016/j.clineuro.2009.01.004. [PubMed: 19200644].

3. Etgen T, Steinich I, Gsottschneider L. Thrombolysis for ischemic stroke in patients with brain tumors. J Stroke Cerebrovasc Dis. 2014;23(2):361-6. doi: 10.1016/j.jstrokecerebrovasdis.2013.05.004. [PubMed: 23763899].

4. Neil W, Ovbiagele B. Intravenous thrombolysis in ischemic stroke patients with intracranial neoplasms: two cases and a literature review. Case Rep Med. 2011;2011:503758. doi:10.1155/2011/503758. [PubMed: 22007235].

5. Rubinshtein R, Jaffe R, Flugelman MY, Karkabi B, Lewis BS. Thrombolysis in patients with a brain tumour. Heart. 2004;90(12):1476. doi: 10.1136/hrt.2003.031013. [PubMed: 15547033]. 
Figure 1. Brain MRI T1Weigthed Image With Gadolinium

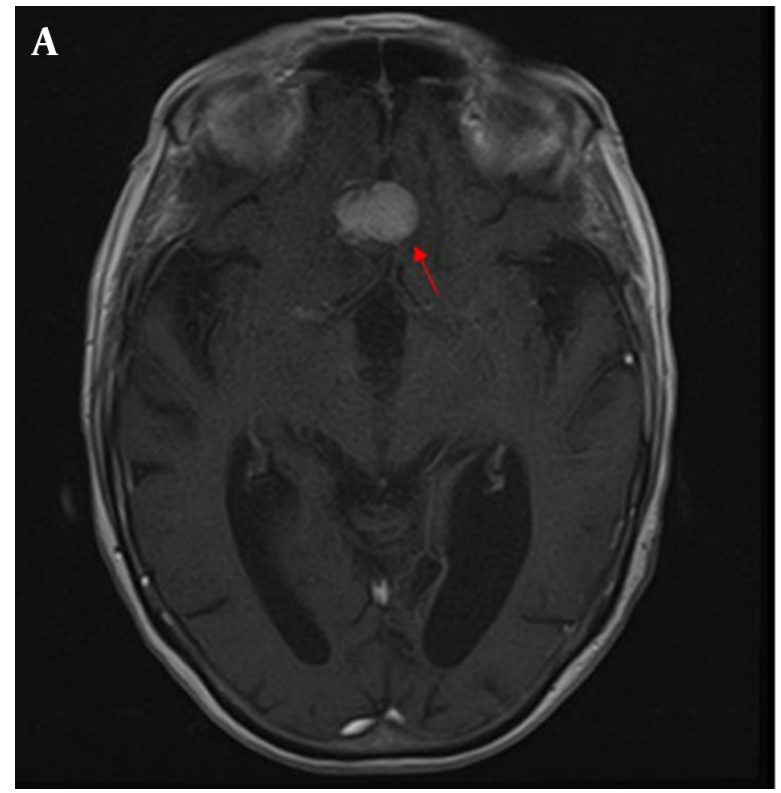

B

A, A $2.2 \mathrm{~cm}$ homogeneous enhancing mass of the planum sphenoidale suggestive of meningioma; B, A $0.9 \mathrm{~cm}$ enhancing nodule in the left frontal convexity, compatible with meningioma. 